\title{
Spectral subtraction-based filter for experimental modal analysis under harmonics excitation force
}

\author{
C. K. E. Nizwan ${ }^{1}$, M. F. Ghazali'1, and A. R. Yusoff ${ }^{2}$ \\ ${ }^{1}$ Faculty of Mechanical and Automotive Engineering, Universiti Malaysia Pahang, 26600 Pekan, Pahang, Malaysia \\ Phone: +6094246217; Fax: +6094246222 \\ 2 Department of Industrial Engineering, College of Engineering, Universiti Malaysia Pahang, 26300 Kuantan, Pahang, Malaysia
}

\begin{abstract}
In modal analysis, measurement of input force and vibration response are crucial to accurately measure the transfer function of the structure. However, under operating condition, the force induced by operating machinery is impossible to be measured due to the sensor placement issue. In this case, the ambient response induced by the operating force should be suppressed to minimize the error in the Frequency Response Function (FRF) calculation. This paper presents the utilization of a modified spectral subtraction filter for ambient suppression. The introduction of effective ambient magnitude in gain function calculation has increased the efficiency of spectral subtraction filter. This parameter is calculated based on the phase information of the reconstructed artificial ambient response. The measurement using EMA was carried out on a motor-driven structure to verify the proposed technique. Two sets of data under shutdown and running condition were recorded to observe the effect of ambient operating force. Under the operating condition, the measured FRF show the non-identical features at operating frequencies as compared to the baseline data. The utilization of filtering process shows the ambient features contained in the transfer function was effectively suppressed. The output of filtering algorithm could provide an alternative option to perform EMA procedure under running condition.
\end{abstract}

ARTICLE HISTORY

Received: 05th May 2021

Revised: $14^{\text {th }}$ June 2021

Accepted: $21^{\text {st }}$ June 2021

KEYWORDS

Condition monitoring;

experimental modal

analysis;

adaptive filter;

harmonics elimination.

\section{INTRODUCTION}

In structure dynamics study, modal parameters provide vital information to define the behaviour of a structure under dynamic loads. Modal analysis technique named experimental modal analysis (EMA) was introduced to deliver reliable system identification algorithms for the determination of the modal parameters of a mechanical structure. This technique is used to identify a system model for the structure based on the measured forces and the vibration response. The modal parameters of the structure are extracted from the identified system model [1].

Experimental Modal Analysis (EMA) utilizes the input force and vibration response measurements to estimate the modal parameters, including natural frequencies, mode shapes, modal damping and the modal participation factors [2]. In the EMA procedure, one or more input forces are induced to the structures and the vibration response are measured at one or several locations to obtain the frequency response function (FRF). The identification of modal parameters is based on the FRF of both the measured input and response signals [3].

In certain cases, the modal analysis procedure could not be applied due to the presence of unmeasurable excitation forces such as the vibration of bridges, offshore platforms, building and during flight test of helicopters and aircraft [4]. Under this condition, the transfer function calculation is not possible to be calculated with the absence or incomplete information of the input forces. In these cases, Operational Modal Analysis (OMA), also denoted as ambient vibration analysis or output-only analysis is one of the possible options to evaluate some of the modal parameters for the structure that are excited randomly by environmental loading conditions [5-8]. OMA technique is a solution to overcome the inability to measure the input forces in EMA by introducing an interesting tool to identify the modal characteristic parameters.

However, the mode shape obtained from the OMA method could not precisely be scaled due to the undetermined modal scaling factor. In case of stiffness and mass properties are to be extracted, scaling is required. Moreover, scaled mode shapes are needed by some vibration-based damage identification methods. Apart from that, the application of OMA requires the excitation of broadband ambient forces to extract all the possible modes. The modal parameters that can be determined from an OMA test can be limited under narrow banded excitation[1].

Impact Synchronous Modal Analysis (ISMA) is one of the processing technique in the EMA procedure to apply the measurement under running condition. This technique utilized the time-averaging method to filter out all the responses contributed by the unaccounted forces, leaving only the response due to the impact hammer [9]. In this case, the response from the unaccounted forces is suppressed even containing the same frequencies as impact response due to inconsistent phase with respect to the impact response. Next, the filtered response will be processed using the EMA procedure and Fast Fourier Transformation (FFT) operation to obtain the transfer function and modal parameter extraction. However, 
the application of ISMA on large structures, where the impact response may be too small in comparison to the operating cyclic loads requires more impacts and may result in non-linearity [9].

The frequency-domain analysis is a very useful tool in analyzing dynamic signals which provide the signal intensity with respect to the frequency spectrum. This signal processing procedure utilized Fourier transform to decompose a time series signal into an infinite or finite number of sinusoids signals with the information of magnitude and phase for each frequency band [10]. On the other hand, the inverse Fourier transform is the process to convert the signal back from the frequency domain to the time domain. These analyses are widely used to manipulate dynamic signals.

The spectral subtraction filter is a method that adopted Fourier analysis to retrieve the power spectrum or magnitude spectrum of a signal found in additive noise by removing the noisy signal spectrum from an approximation of the average noise spectrum [11]. The two main components for spectral subtraction is calculating the spectral of the background noise and remove the noisy spectral from the noisy signal. The spectral subtraction works based on the expectation that the spectral component of the input signal is gained from the addition of the cleared signal with the noise [12]. Spectral subtraction has been shown to be an efficient method for minimizing ambient acoustic noise and thereby improving the intelligibility and efficiency of digitally compressed speech. It is demonstrated that spectral subtraction can be applied using a nonstationary, multiplicative frequency-domain filter that varies with the time changing spectral characteristics of the voice [13].

This paper focuses on the development of a modified spectral subtraction filter algorithm to suppress the ambient features contained in the measured vibration response under operating condition. The impact responses measured from the EMA procedure is superposition with the response that induced by the operating machinery. In this situation, the responses contributed by the ambient operating force require the filtering process due to unknown information for the force quantities which prerequisite in transfer function calculation. The algorithm utilized the modified spectral subtraction to suppress the ambient response. In this method, the phase of the vibration spectrum required a reallocation process to minimize the phase difference for the post-filter signals. Then, a clean vibration response from impact forced is extracted based on the ratio between estimated ambient with measured impact response.

\section{EXPERIMENTAL SETUP}

This section will discuss the measurement process to obtain the dynamic characteristics of a motor-driven structure through experimental modal analysis (EMA). The rig structure consists of a two-layer platform with a motor speed controller and an electric motor attached to the upper platform of the rig structure as shown in Figure 1. The measurements were applied under two different conditions which are shut down and running condition. In this EMA procedure, the artificial impact force induced into the structure using the impact hammer and the vibration responses were measured using a tri-axial accelerometer. The sampling frequency and block size are selected at $2048 \mathrm{~Hz}$ and 4096 respectively. In running condition, the ambient forces were induced by an unbalanced mass attached to the disk and speed controller cooling fan.

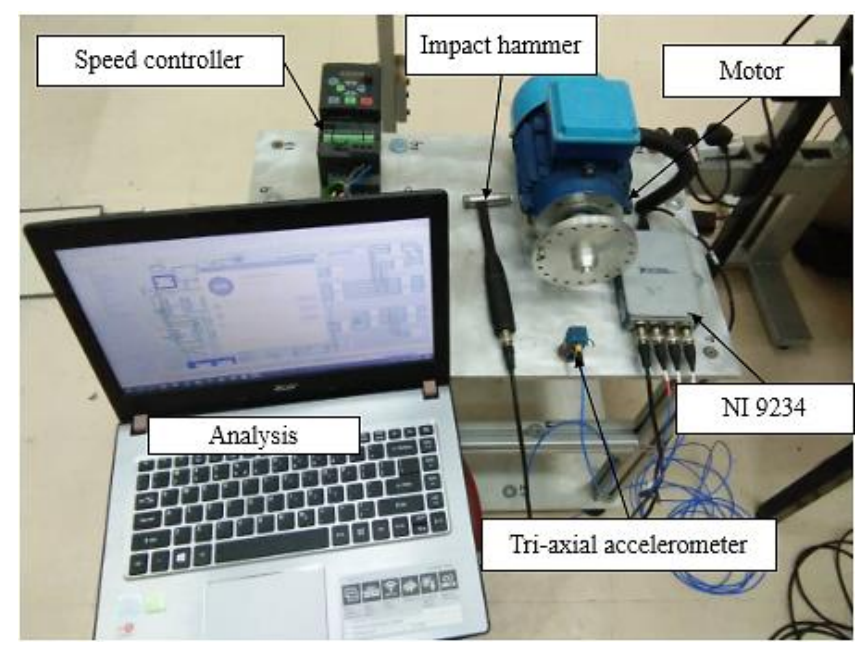

Figure 1. Experimental setup for EMA

The frequency response function (FRF) is a basic principle of measurement for obtaining the mechanical structural dynamic characteristics of a structure [14]. It is also known as a transfer function and is denoted by the frequency domain. The equation for the transfer function is obtained from the linear mechanical system. The equation for the transfer function [15] is represented by Eq. (1).

$$
H(\omega)=\frac{X(\omega)}{F(\omega)}
$$


Where $H(\omega)$ is a transfer function, $X(\omega)$ is an output response and $F(\omega)$ is an input force. In this study, DASYLab software is utilized to record all the data and perform FRF calculation based on the transfer function module. Next, the natural frequencies, mode shapes and damping value are estimated based on the curve fitting process using MEScope software. All the vibration responses were recorded for the next filtering process.

\section{DEVELOPMENT OF AMBIENT SUPPRESSION FILTER}

In Experimental Modal Analysis, the Frequency Response Function (FRF) is calculated based on cross-correlation and autocorrelation of measured input force and vibration response. However, if EMA was performed with the presence of ambient forces, the measured vibration response can be expressed as a superposition of impact response and ambient responses as shown in Eq. (2) [9]. The calculation of FRF for the system is not possible to proceed due to unavailable information of ambient forces. As a solution, the measured vibration response requires post analysis to suppress the ambient effect contains in the initial measurement.

$$
X(\omega)=\left[H_{1}(\omega)^{*} F_{1}\right]+\left[H_{2}(\omega) * F_{2}\right]+\left[H_{3}(\omega)^{*} F_{3}\right]+\cdots
$$

This research utilized the spectrum subtraction filter [13] to suppress the ambient effect from the in-operation impact responses measurement. This filtering technique was developed based on the ratio between deviations of measured impact response with ambient in term of the frequency spectrum. Vibration response due to impact and ambient, $x_{\text {total }}$ and ambient only, $x_{\text {ambient }}$ are required input to perform the filtering proses using SS filter.

The concept of this filter is to suppress the spectrum magnitude of the ambient, $S_{\text {ambient }}(\omega)$ from the in-operation impact response, $S_{\text {total }}(\omega)$ based on amplitude normalization with the gain function, $G(\omega)$. $S_{\text {ambient }}(\omega)$ and $S_{\text {total }}(\omega)$ is the output Fast Fourier transform of the impact and ambient response under operation condition as shown in Eq. (3) and Eq. (4). Eq. (5) shows the equation for gain function and the output of the filtering process, Extracted Impact Spectrum (EIS) is expressed in Eq. (6).

$$
\begin{gathered}
S_{\text {total }}(k)=\sum_{j=1}^{n} x_{\text {total }}(j) e^{\frac{-2 \pi i(j-1)(k-1)}{n}} \\
S_{\text {ambient }}(k)=\sum_{j=1}^{n} x_{\text {ambient }}(j) e^{\frac{-2 \pi i(j-1)(k-1)}{n}} \\
G(\omega)=\frac{\left|S_{\text {total }}(\omega)\right|-\left|S_{\text {Ambient }}(\omega)\right|}{\left|S_{\text {total }}(\omega)\right|} \\
E I S=\frac{\left|S_{\text {total }}\right|-\left|S_{\text {Ambient }}\right|}{\left|S_{\text {total }}\right|} \cdot S_{\text {total }}
\end{gathered}
$$

This process produced a normalized spectrum in term of magnitude and the Inverse Fourier Transform is utilized to convert EIS into the time-domain signal, $x_{\text {outpu }}$. A sample of data consists of a harmonic signal, $x_{\text {ambient }}$ and the combination of harmonic and transient harmonic $\left(x_{\text {impact }}\right), x_{\text {total }}$ is used to verify the efficiency of the filter. In this simulation, a damped single degree of freedom response was used with the excitation of harmonic force. The transient response is represented as $x_{\text {impact }}$ and $x_{\text {ambient }}$ is referred to as the steady-state response due to harmonic excitation. Both of the signals were superpositioned as $x_{\text {total }}$ to simulate the transient response under harmonic excitation force.

The filtering process will be carried out under two different condition of ambient response i.e. synchronous phase and non-synchronous phase. All of the signals used for the synchronous phase condition are shown in Figure 2 and Eq. (7) shows the mathematical equation of the corresponded signals.

$$
\begin{aligned}
& x_{\text {impact }}(\mathrm{t})=\mathrm{e}^{-2 \mathrm{t}} 10 \sin (10 \pi \mathrm{t}) \\
& x_{\text {ambient }}(\mathrm{t})=2 \sin (10 \pi \mathrm{t}) \\
& x_{\text {total }}=x_{\text {impact }}+x_{\text {ambient }}
\end{aligned}
$$




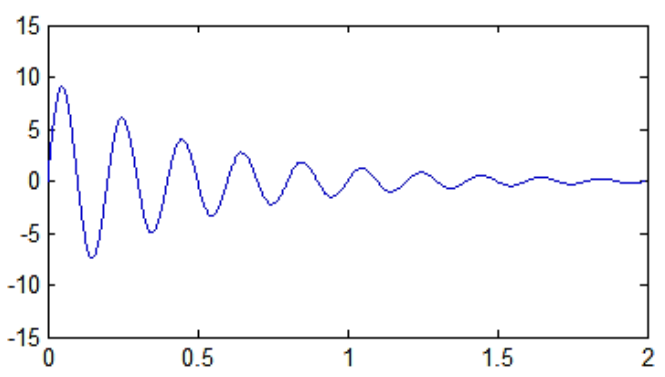

(a)

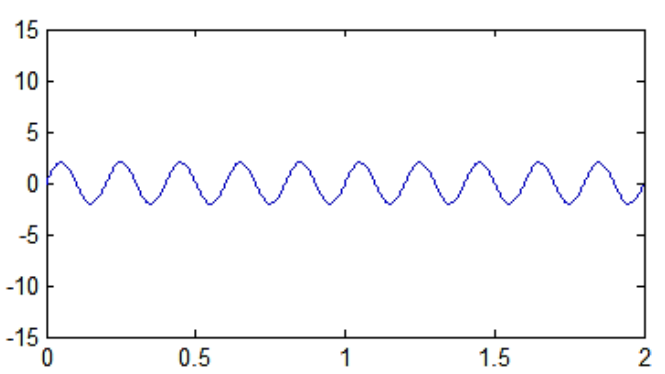

(b)

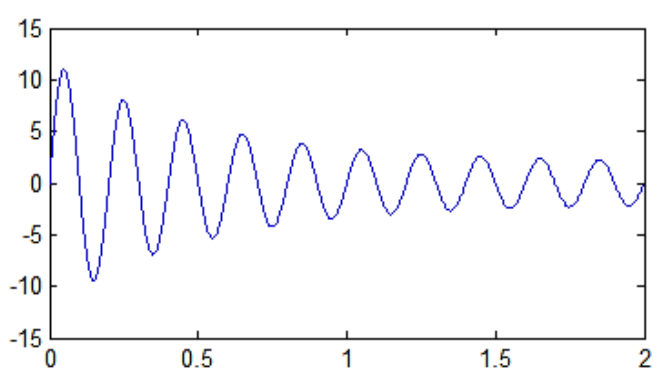

(c)

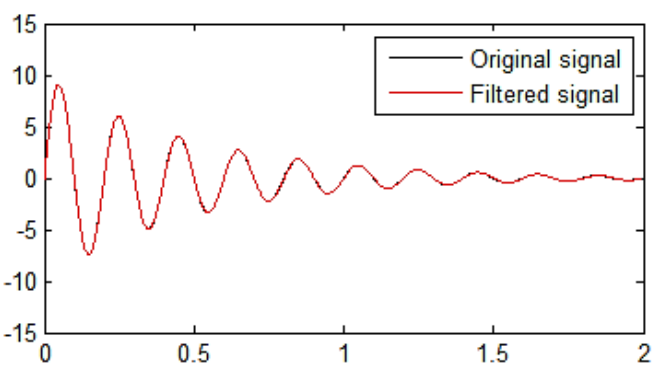

(d)

Figure 2. (a) Impact signal, $x_{\text {impact }}$ (b) Harmonic ambient, $x_{\text {ambient }}$ (c) Impact + harmonic, $x_{\text {total }}$ (d) Overlaid Impact signal, $x_{\text {impact }}$ and Filtered signal, $x_{\text {output }}$

For this simulation, both $x_{\text {ambient }}$ and $x_{\text {impact }}$ have equivalent frequency and phase but different amplitudes. In comparison to Impact Modal Analysis, $x_{\text {total }}$ and $x_{\text {ambient }}$ represent the response of impact under harmonic excitation and ambient response respectively. For this situation, the output of the filtering process in Figure 2(d) shows the filtered signal is identical to the transient signal which indicated that this filter successfully eliminates the harmonic ambient.

Next, the same procedure was repeated for the case of non-synchronous ambient. The ambient for this case was set as $45^{\circ}$ out of phase as represented in Eq. (8).

$$
x_{\text {ambient }}(t)=2 \sin (10 \pi t+\pi / 4)
$$

However, for non-synchronous $x_{\text {ambient }}$ and $x_{\text {impact }}$, the output of filtering proses shows the filtered signal has a different phase and the suppressed amplitude is not as efficient as synchronous ambient. The detail of these signals is illustrated in Figure 3. This phenomenon happened because the non-synchronous $x_{\text {ambient }}$ and $x_{\text {impact }}$ signal will affect the phase of $x_{\text {total }}$ [16], and this filter is designed to suppress the amplitude of the spectrum without considering the phase. Therefore, the phase of the filtered signal is corresponding to the $x_{\text {total }}$ and not the expected $x_{\text {impact }}$. 
Hence, phase reallocation is vital for $x_{\text {total }}$ as a pre-process before performing the filtering process to maintain the original shape of the impact response. In the vector diagram as shown in Figure 3, the phase of impact response can be retrieved if the information of magnitude and phase of the ambient signal is known. The phases of impact vectors were calculated based on the following equation (Eq. (9)).

$$
\theta_{n e w, i}=\tan ^{-1}\left(\frac{\operatorname{Imaginary}\left(\vec{X}_{\text {total }, i}-\vec{X}_{\text {ambient }, i}\right)}{\operatorname{Real}\left(\vec{X}_{\text {total }, i}-\vec{X}_{\text {ambient }, i}\right)}\right)
$$

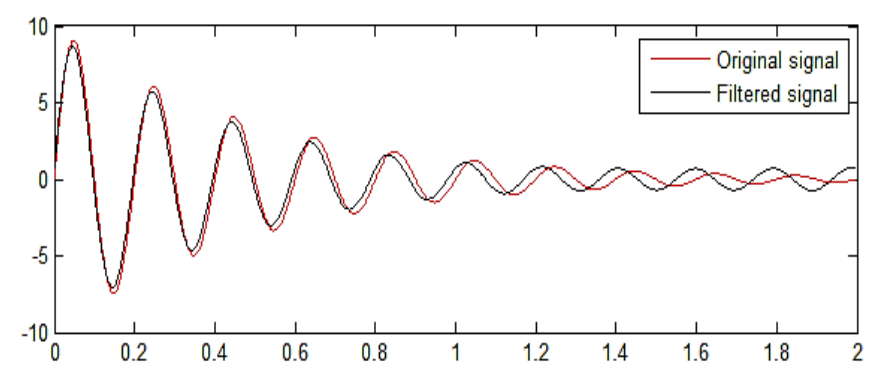

Figure 3. Comparison between the filtered and original signal for non-synchronous harmonic ambient

Then, the $S_{\text {total }}$ vectors were rotated according to the $\theta_{\text {new }, i}$ as illustrated in Figure 4 . The purpose of the process is to minimize the change of phase in impact response due to the presence of ambient. In order to increase the accuracy of signal amplitude, the magnitude of ambient in SDR calculation is considering the effective magnitude in $S_{\text {total }}$ direction, which equal to:

$$
\left|S_{\text {effective }}\right|=\left|S_{\text {ambient }}\right| \cos \left(\theta_{\text {new }}-\theta_{\text {ambient }}\right)
$$

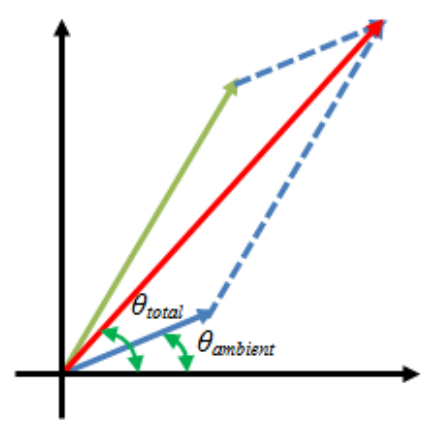

(a)

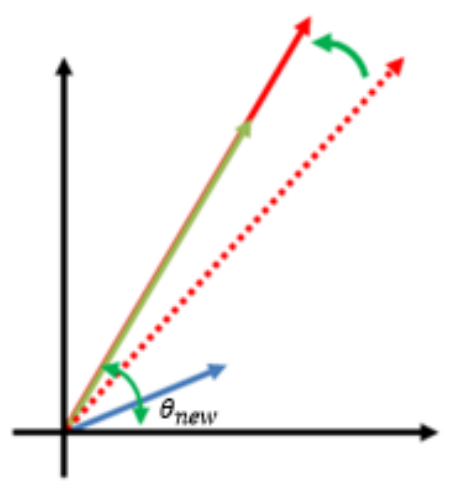

(b)

Figure 4. (a) Vector of ambient and impact spectrum and (b) Rotational transform of the measured spectrum

The phase reallocation was then applied for the impact signal with non-synchronous ambient. After filtering proses using spectrum deviation filter, the output of the phase reallocation signal shows the filtered signal has successfully retained the phase of the original signal compare to the filtering process without phase reallocation. Figure 5 shows the overlaid of the filtered signal with the original signal.

In real measurement, this technique required the information of ambient response in the period of recorded impact response, which not possible to accurately measure. For this case, the artificial ambient responses were used to represent 
the desired ambient responses. The artificial ambient responses were generated based on the vectors of the Fourier Transform of the successive block of impact response [17]. The sample for this analysis is from the ambient response generated by a motor-driven structure. The aim is to reproduce an artificial signal for the first block of data based on the information from the second block of data (inside the rectangular shape). Data from the second block was mirrored and transformed into the frequency domain using the FFT algorithm. The Inverse Fourier Transform algorithm was utilized to reconstruct the signal in a longer form which is twice from the original sample. Next, the second half of the reconstructed data was mirrored in order to reproduce the desired artificial ambient. Figure 6 shows the graphical process of generating an artificial signal for the sample of ambient response. The artificial signal generated through this method shows high similarity with the measured ambient response shown in Figure 7.

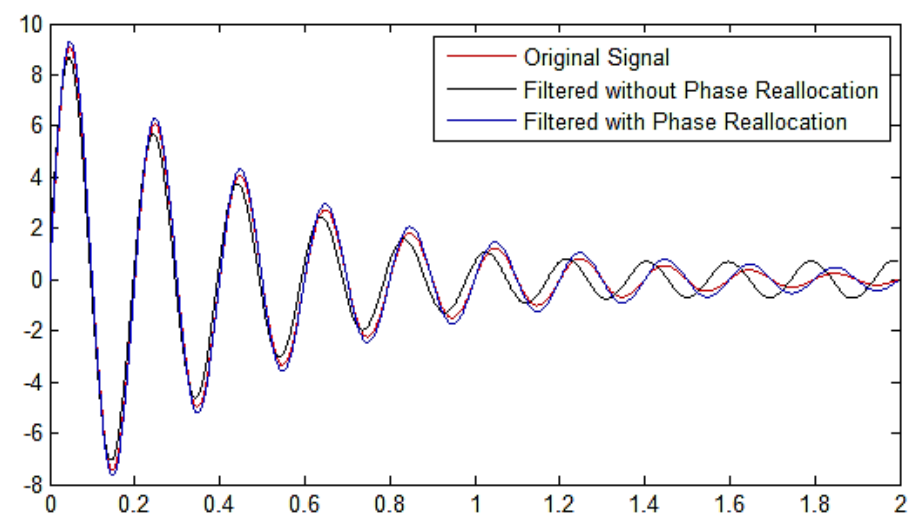

Figure 5. Comparison between filtered signal with and without phase reallocation

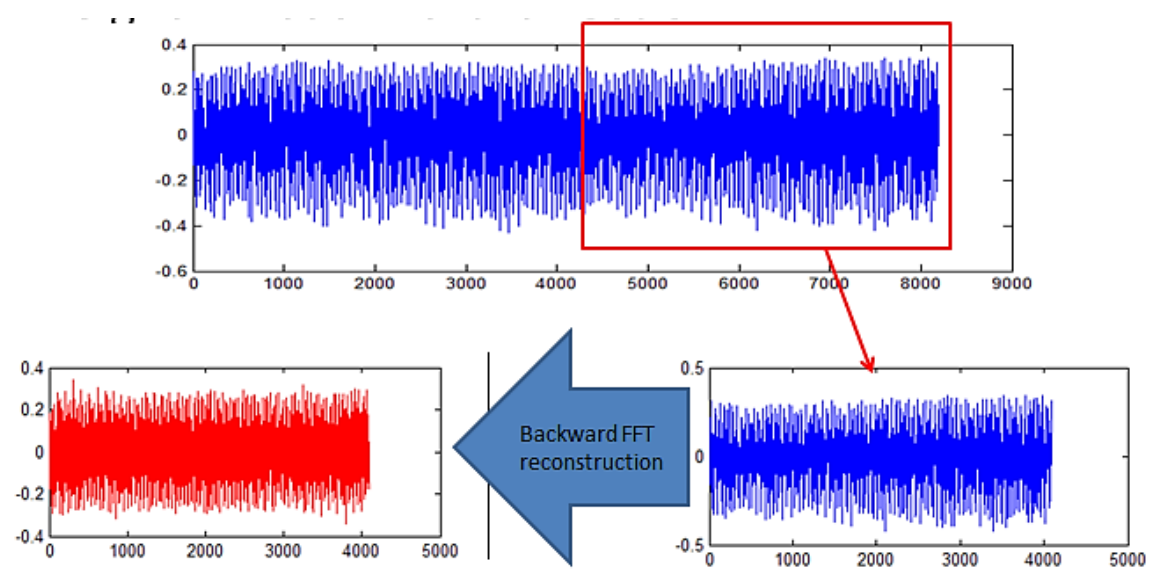

Figure 6. The process of generating the artificial ambient response

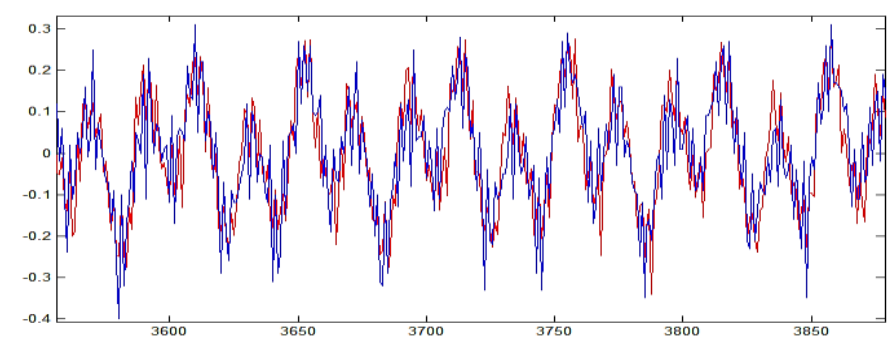

Figure 7. Overlapping of artificial and measured ambient response

This filtering technique will be utilized on the experimental modal analysis procedure under the operating condition to suppress the ambient components. The procedure requires the information of impact response under harmonic excitation and ambient characteristics in term of magnitude and phase. 


\section{RESULT AND DISCUSSION}

\section{Experimental Modal Analysis}

The curve fitting method is applied to the frequency response function (FRF) to determine the mode shapes representing the natural frequencies of the rig structure [18]. The global polynomial is used to form the frequency domain to help the curve fitting method in determining the frequency response function (FRF) [19]. The horizontal axis illustrates the frequency of the structure in Hertz $(\mathrm{Hz})$ while the vertical axis exhibits the magnitude of vibration response. For this analysis, two conditions are being studied. The experimental modal analysis (EMA) was carried out onto the rig structure in a condition where the electric motor is completely shut down and the other condition where the electric motor is operated at $30 \mathrm{~Hz}$ and $40 \mathrm{~Hz}$. The data obtained from the experiment is analysed and shown in the table for comparison. As shown in Figure 8, the frequency response function (FRF) data for the shutdown condition is shown in an overlay format. From the data, the modal parameter information is obtained and gathered in Table 1. For the shutdown condition, five mode-shapes have been determined from the frequency response function (FRF) by using the multivariate mode indicator function (MMIF). Even though there are several natural frequencies are located in the data, they could be ignored as they only have a small amplitude and some of them are not being excited [20].

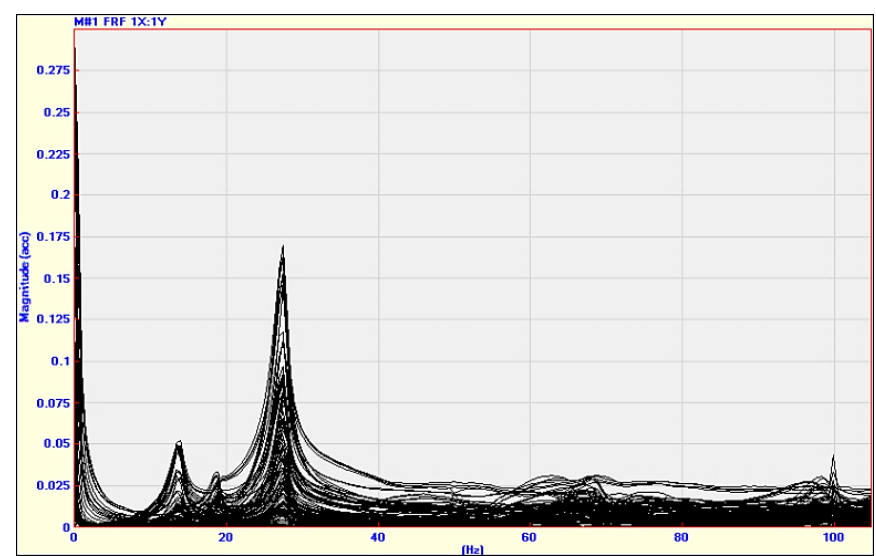

Figure 8. Overlaid frequency response function (FRF) for shutdown condition

Table 1. Modal parameter for baseline data

\begin{tabular}{ccc}
\hline Mode & Frequency $(\mathbf{H z})$ & Damping $(\%)$ \\
\hline 1 & 13.7 & 5.40 \\
2 & 18.9 & 3.05 \\
3 & 27.4 & 3.03 \\
4 & 67.7 & 3.63 \\
5 & 100.0 & 0.318 \\
\hline
\end{tabular}

As shown in Figure 9, the frequency response function (FRF) data for the operating condition is shown in an overlay format. The overlaid FRF plot for operating condition shows the presence of three peaks frequency at $40 \mathrm{~Hz}, 80 \mathrm{~Hz}$ and $100 \mathrm{~Hz}$ which represent the rotor speed, two times of rotor speed and the cooling fan operating speed respectively. The vibration created by the electric motor is called a noisy signal which is needed to be filtered to get the clean signal.

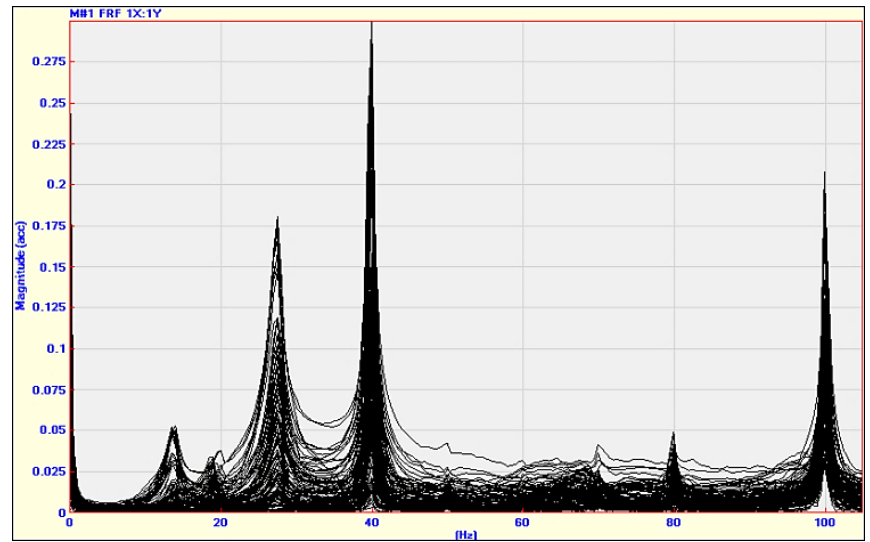

Figure 9. Frequency response function (FRF) for operating condition 


\section{Filtering Algorithm for Ambient Suppression}

In this research, the vibration responses acquired from the measurement process under running conditions consist of two main clusters i.e. response from impact force and response from the ambient force. Due to the unknown value for the forces induced by rotating equipment, the measured vibration response required a filtering process to suppress the response due to the ambient operating forced.

The modified spectral subtraction filter was utilized to suppress the ambient response in EMA for a motor-driven structure. The time-domain plot for impact responses under shut-down condition, running and after filtered are overlaid in Figure 10. From the time domain plot, it is clearly seen that the ambient is completely being filtered out by the modified spectral subtraction filter. The proposed method has successfully suppressed the ambient without affecting any useful data from the signal which is very important to be preserved in order to find the modal parameter of the structure.

The previous result on experimental modal analysis shows, under the harmonics ambient excitation, the natural mode which closes to the operating frequency will be affected especially on the peak value of natural frequency and mode shape. In this case, the transfer function of the filtered signal will be compared with the condition when the system is shut down. One of the samples of the Frequency Response Function (FRF) plot in Figure 11 shows at the operating frequency of $40 \mathrm{~Hz}$, the peak frequency for filtered signal without phase reallocation are over suppressed compare to the shutdown condition. This situation is caused by the effective ambient magnitude was not accurately calculate due to a lack of information in the ambient phase. However, with phase reallocation, the peak frequency was restored close to the shutdown condition and the overall FRF plot shows high similarity with baseline data.
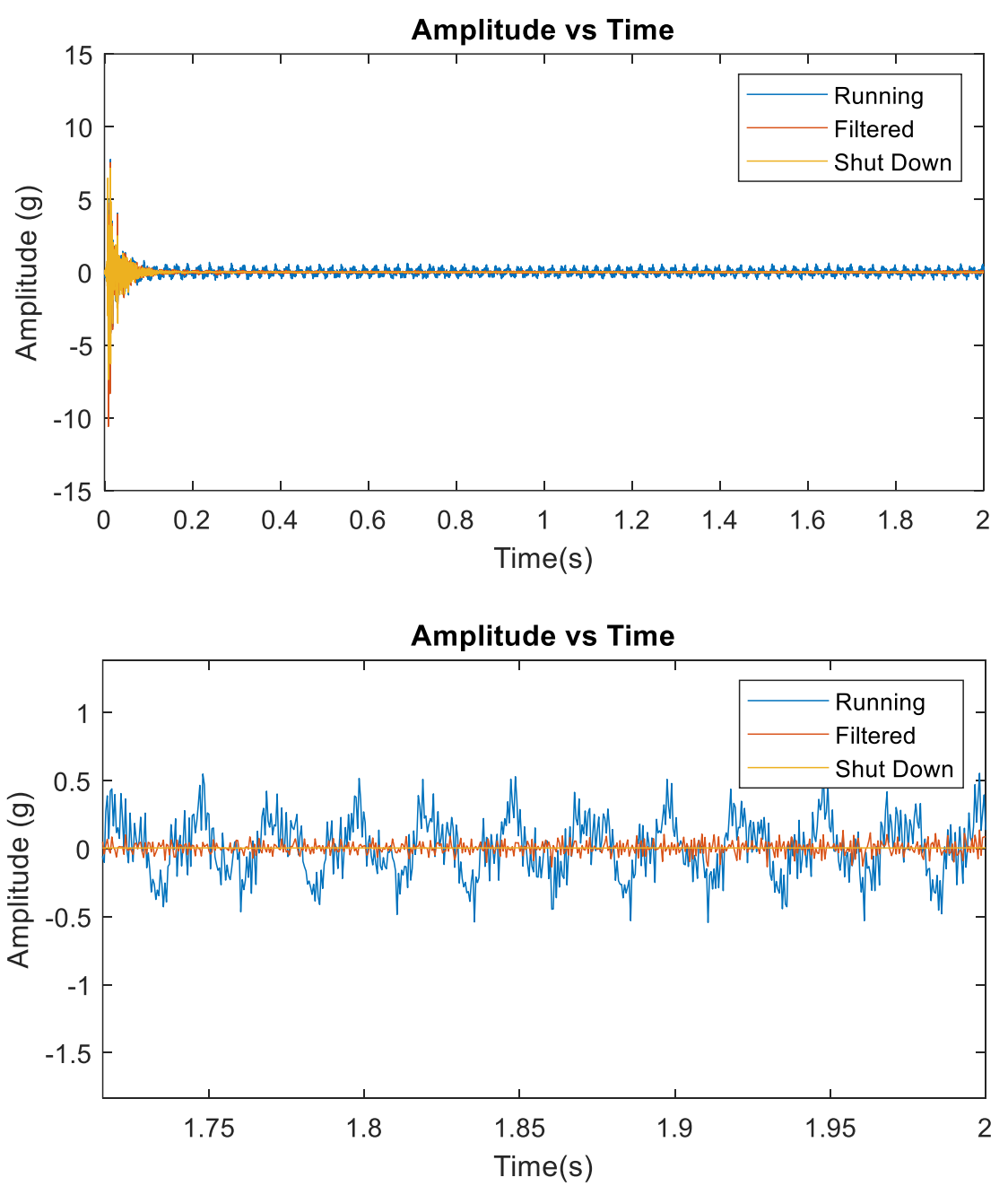

Figure 10. Time-domain plot for impact response 


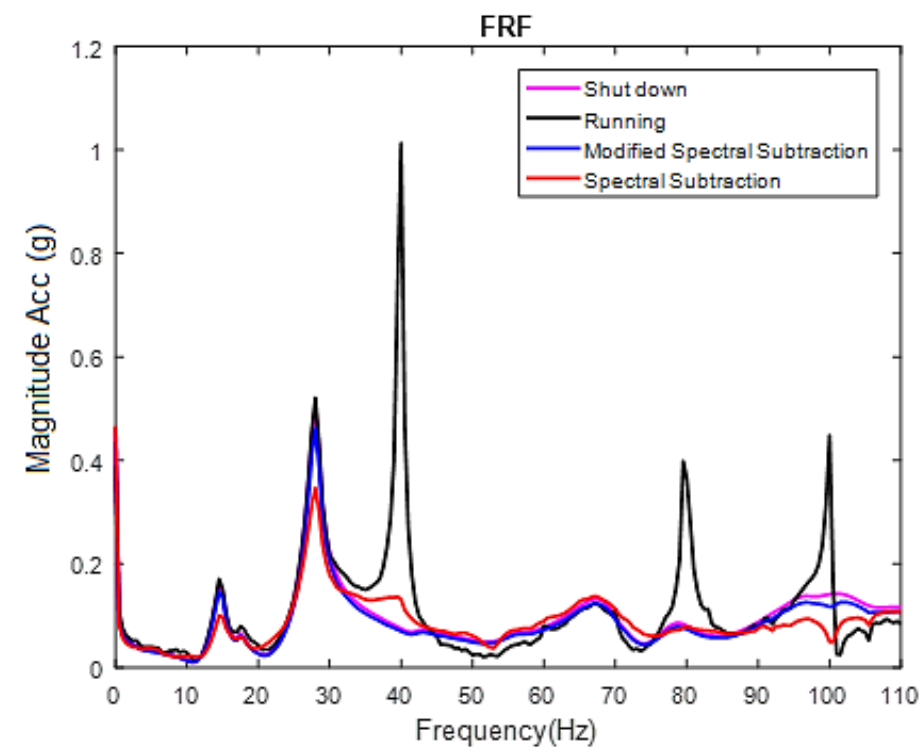

Figure 11. Comparison FRF plot between shut down and filtered signal

Modal assurance criterion or widely known as MAC is a type of analytical measurement that measures the changes in the mode shapes. Normally, the sensitivity of the MAC is determined by the variation in the mode shapes. MAC works better with large differences in the mode shapes compared to the small differences. In the modal analysis, MAC is used to compare the mode shapes that are obtained from both mathematical and experimental model. The correlation of the mode shapes is characterized by the value of 0 till 1 . The value that is closer to zero means that the mode shapes is uncorrelated whereas the value that is closer to one means that the mode shapes is correlated. It is also stated the minimum value between two correlated mode shapes is 0.9 [21]. As shown in Table 2, the modal assurance criterion (MAC) is presented in 2 Dimension distributions. From the distributions, it is clearly seen that the entire mode shapes are correlated as all of them are achieved the minimum value of the correlation.

Table 2. Modal assurance criterion (MAC)

\begin{tabular}{|c|c|c|c|c|c|c|c|}
\hline \multirow{2}{*}{ Mode } & \multicolumn{2}{|c|}{ After Filter } & $\mathbf{1}$ & $\mathbf{2}$ & $\mathbf{3}$ & $\mathbf{4}$ & $\mathbf{5}$ \\
\hline \multirow{2}{*}{ Base-line } & \multicolumn{2}{|c|}{ Natural Frequency } & 13.6 & 19.0 & 27.4 & 67.7 & 100.0 \\
\cline { 2 - 8 } & & Damping (\%) & 5.48 & 3.07 & 3.03 & 3.69 & 0.302 \\
\hline 1 & 13.7 & 5.4 & 1.000 & 0.001 & 0.007 & 0.007 & 0.008 \\
\hline 2 & 18.9 & 3.05 & 0.002 & 0.997 & 0.002 & 0.018 & 0.033 \\
\hline 3 & 27.4 & 3.03 & 0.007 & 0.003 & 1.000 & 0.001 & 0.003 \\
\hline 4 & 67.7 & 3.63 & 0.004 & 0.017 & 0.001 & 0.939 & 0.013 \\
\hline 5 & 100.0 & 0.318 & 0.009 & 0.034 & 0.003 & 0.009 & 0.914 \\
\hline
\end{tabular}

AutoMAC is a quantitative comparison of a set of data that consists of both mode shapes that are being studied in a single datasheet [20]. Values between 0 and 1.0 are equivalent to the extent to which the mode shapes correlate. Based on Table 2, mode shape 1 and mode shape 3 are completely correlated at the value of 1.0. Mode shape 4 showed the lowest value at 0.939 but it is still in the range value for the mode shape to be considered as correlate according to the modal assurance criterion (MAC). The values shown by AutoMAC is similar to the values shown by the bar chart in MAC.

\section{CONCLUSIONS}

This paper discusses the study of an ambient suppressing technique using a modified spectral subtraction filter. This filtering method improvised the gain function in the spectral subtraction filter. The gain function is determined from the effective ambient magnitude, which calculates based on the relative magnitude between ambient and total response. The filtering process retained the residual part of the measured contaminated impact response after suppressing the range of effective ambient magnitude to produce a clean impact response.

In this study, the EMA procedure is executed on a motor-driven structure to obtain the transfer function of the system under two different conditions i.e. shut down and in operation. In comparison, the transfer function obtained from the in 
operation measurement shows the frequency components at operating speed were affected with respect to the shutdown condition. Then, the filtering algorithm is applied to the in-operation vibration response to suppress the ambient features. An artificial ambient is reconstructed to replicate the ambient signatures in the period of the triggered impact response. This process is purposely to retrieve the phase information of the ambient response for effective ambient magnitude calculation. With the complimentary of effective ambient magnitude, the spectral subtraction filter shows a significant improvement in suppressing the ambient features as compared with conventional spectral subtraction. The findings indicate that the modified Spectrum Subtraction filter can be used to develop an effective filtering algorithm for EMA procedure under ambient operating force.

\section{ACKNOWLEDGMENTS}

The authors would like to express their gratitude to Universiti Malaysia Pahang through the fund of FRGS/1/2019/TK03/UMP/03/6 (RDU1901192), for supporting these research activities.

\section{REFERENCES}

[1] E. Reynders and G. De Roeck, "Reference-based combined deterministic-stochastic subspace identification for experimental and operational modal analysis," Mech. Syst. Signal Process., vol. 22, no. 3, pp. 617-637, 2008, doi: 10.1016/j.ymssp.2007.09.004.

[2] N. M. M. Maia and J. M. M. e Silva, Theoretical and experimental modal analysis. Research Studies Press, 1997.

[3] F. H. Jackson, “Analytical methods in vibrations," Electron. Power, vol. 13, no. 12, p. 480, 1967, doi: 10.1049/ep.1967.0353.

[4] J. L. Dion, I. Tawfiq, and G. Chevallier, "Harmonic component detection: Optimized Spectral Kurtosis for operational modal analysis,” Mech. Syst. Signal Process., vol. 26, no. 1, pp. 24-33, 2012, doi: 10.1016/j.ymssp.2011.07.009.

[5] T. Carne, J. Lauffer, A. J. Gomez, and H. Benjannet, "Modal testing an immense flexible structure using natural and artificial excitation," Int. J. Anal. Exp. Modal Anal., vol. 3, pp. 117-122, Jan. 1988.

[6] J. P. Lauffer, T. G. Carrie, and A. J. Gomez, "Modal survey of the 34-m test bed turbine," Sandia Natl. Lab. Intern. Memo to PC Klimas Div., vol. 6225, 1988.

[7] P. Mohanty and D. J. Rixen, "Operational modal analysis in the presence of harmonic excitation,” J. Sound Vib., vol. 270 , no. 1-2, pp. 93-109, 2004, doi: 10.1016/S0022-460X(03)00485-1.

[8] R. Tarinejad, K. Falsafian, M. T. Aalami, and M. T. Ahmadi, "Modal identification of Karun IV arch dam based on ambient vibration tests and seismic responses," J. Vibroengineering, vol. 18, no. 6, pp. 3869-3880, 2016, doi: 10.21595/jve.2016.16989.

[9] A. G. A. Rahman, O. Z. Chao, and Z. Ismail, "Effectiveness of impact-synchronous time averaging in determination of dynamic characteristics of a rotor dynamic system," Meas. J. Int. Meas. Confed., vol. 44, no. 1, pp. 34-45, 2011, doi: 10.1016/j.measurement.2010.09.005.

[10] R. L. Allen and D. Mills, Signal analysis. John Wiley \& Sons, 2004.

[11] N. Upadhyay and A. Karmakar, "Speech enhancement using spectral subtraction-type algorithms: A comparison and simulation study," Procedia Comput. Sci., vol. 54, pp. 574-584, 2015, doi: 10.1016/j.procs.2015.06.066.

[12] B. Nikita, C. Prajakta, D. Priyanka, I. Nisha, and R. Amarsinh, "Speech enhancement based on spectral subtraction involving magnitude and phase components," Int. Res. J. Eng. Technol., vol. 4, no. 3, pp. 2483-2488, 2017.

[13] S. F. Boll, "Suppression of acoustic noise in speech using spectral subtraction," IEEE Trans. Acoust., vol. 27, no. 2, pp. 113120, 1979, doi: 10.1109/TASSP.1979.1163209.

[14] B. J. Schwarz and M. H. Richardson, "Experimental modal analysis," CSI Reliab. week, vol. 35, no. 1, pp. 1-12, 1999.

[15] W. Pawlowski, L. Kaczmarek, and P. Louda, "Theoretical and experimental modal analysis of the cylinder unit filled with PUR foam," Eksploat. i Niezawodn., vol. 18, no. 3, 2016.

[16] T. Kleinschmidt, S. Sridharan, and M. Mason, "The use of phase in complex spectrum subtraction for robust speech recognition,” Comput. Speech Lang., vol. 25, no. 3, pp. 585-600, 2011, doi: 10.1016/j.csl.2010.09.001.

[17] M. Hayes, J. Lim, and A. Oppenheim, "Signal reconstruction from phase or magnitude," IEEE Trans. Acoust., vol. 28, no. 6, pp. 672-680, 1980.

[18] M. N. Jayashankar, "Output only modal analysis of engineering structures." International Journal of Engineering Research \& Technology, vol. 4, no. 11, pp.301-304, 2015.

[19] J. Mahowald, S. Maas, F. Scherbaum, D. Waldmann, and A. Zürbes, "Dynamische Methoden zur Schadensidentifikation an einer kontinuierlich geschädigten vorgespannten Zweifeldbrücke," VDI-Berichte 2160, pp. 247-258, 2012.

[20] M. L. Chandravanshi and A. K. Mukhopadhyay, "Modal analysis of structural vibration," in ASME International Mechanical Engineering Congress and Exposition, 2013, vol. 56437, p. V014T15A052.

[21] M. Pastor, M. Binda, and T. Harčarik, "Modal assurance criterion," Procedia Eng., vol. 48, pp. 543-548, 2012, doi: 10.1016/j.proeng.2012.09.551. 